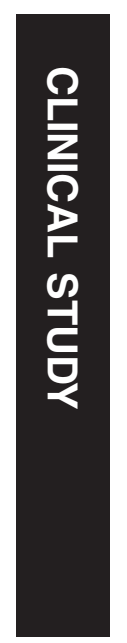

\title{
Measures of socioeconomic status and self-reported glaucoma in the UK Biobank cohort
}

${ }^{1}$ Division of Genetics and Epidemiology, NIHR Biomedical Research Centre, Moorfields Eye Hospital and UCL Institute of Ophthalmology, London, UK

${ }^{2}$ Department of Public Health and Primary Care, University of Cambridge, Cambridge, UK

${ }^{3}$ Singapore Eye Research Institute, Singapore, Singapore

${ }^{4}$ Discipline of Clinical Ophthalmology and Eye Health, University of Sydney, Sydney, NSW, Australia

${ }^{5}$ Members of the UK Biobank Eye and Vision Consortium are listed before References

Correspondence:

PJ Foster, Division of Genetics and Epidemiology, UCL Institute of

Ophthalmology, 11-43 Bath Street, London EC1V 9EL, UK

Tel/Fax: +44 (0)20 7608 6899.

E-mail P.foster@ucl.ac.uk

Received: 19 May 2015 Accepted in revised form: 20 July 2015

Published online:

28 August 2015

\begin{abstract}
Purpose To determine ocular, demographic, and socioeconomic associations with selfreported glaucoma in the UK Biobank. Methods Biobank is a study of UK residents aged 40-69 years registered with the National Health Service. Data were collected on visual acuity, intraocular pressure (IOP), corneal biomechanics, and questionnaire from 112690 participants. Relationships between ocular, demographic, and socioeconomic variables with reported diagnosis of glaucoma were examined. Results In all, $1916(1.7 \%)$ people in UK Biobank reported glaucoma diagnosis. Participants reporting glaucoma were more likely to be older (mean 61.4 vs 56.7 years, $P<0.001)$ and male $(2.1 \%$ vs $1.4 \%, P=0.001)$. The rate of reported glaucoma was significantly higher in Black $(3.28 \%, P<0.001)$ and Asian $(2.14 \%, P=0.009)$ participants compared with White participants $(1.62 \%$, reference). Cases of reported glaucoma had a higher mean IOP (18 $\mathrm{mm} \mathrm{Hg}$ both eyes, $P<0.001)$, lower corneal hysteresis $(9.96$ right eye, 9.89 left eye, $P<0.001$ ), and lower visual acuity (0.09 logMAR right eye, 0.08 logMAR left eye, $P<0.001)$ compared with those without $(16 \mathrm{~mm} \mathrm{Hg}$ both eyes, hysteresis 10.67 right eye, 10.63 left eye, 0.03 logMAR right eye, 0.02 logMAR left eye). The mean Townsend deprivation index was -0.72 for those reporting glaucoma and -0.95 for those without $(P<0.001)$, indicating greater relative deprivation in those reporting glaucoma. Multivariable logistic regression showed that people in the lowest income group $(<£ 18000 /$ year) were significantly more likely to report a diagnosis of glaucoma compared with any other income level $(P<0.01)$. We observed increasing glaucoma risk across the full range of income categories, with highest risk among those of lowest income, and no evidence of a threshold effect.
\end{abstract}

Y Shweikh ${ }^{1}$, F Ko ${ }^{1}$, MPY Chan ${ }^{1}$, PJ Patel', Z Muthy', PT Khaw ${ }^{1}$, J Yip ${ }^{1,2}$, N Strouthidis 1,3,4 and PJ Foster ${ }^{1}$ on behalf of the UK Biobank Eye and Vision Consortium ${ }^{5}$

Conclusions In a large UK cohort, individuals reporting glaucoma had more adverse socioeconomic characteristics. Study of the mechanisms explaining these effects may aid our understanding of health inequality and will help inform public health interventions. Eye (2015) 29, 1360-1367; doi:10.1038/eye.2015.157; published online 28 August 2015

Introduction

Glaucoma is the second commonest cause of blindness worldwide, and the leading cause of medically and surgically irreversible blindness. ${ }^{1}$ In UK, it is the second commonest cause of severe sight impairment registration. ${ }^{2}$

Prevalence of glaucoma increases exponentially with age, with no clear upper limit to the increase in prevalence with age. ${ }^{3}$ As a chronic, age-related disease, current care strategies require regular monitoring of established cases and high-risk suspects. The increase in life expectancy in the UK, together with increasing referral of early cases and suspects has seen $7-8 \%$ per year growth in demand for glaucoma service appointments at Moorfields Eye Hospital. ${ }^{4}$ National Institute for Health and Care Excellence (NICE) guidelines for the diagnosis and management of chronic open-angle glaucoma and ocular hypertension in 2009 (NICE, 2009) led to significant increases in referral numbers across the NHS. 5,6

Epidemiological data form a core part of the evidence base for clinical decision-making, informing judgements about risk of disease according to age, sex, racial origin, systemic and ocular risk factors. Currently, data on the epidemiology of glaucoma in people of European origin are overwhelmingly derived from studies carried out in the United States, Australia, and the Netherlands. ${ }^{7-11}$ Prevalence and risk factor studies from the UK at the 
population level are few and based on work carried out in the 1960 s. ${ }^{12,13}$ More recently, the EPIC Norfolk study ${ }^{14}$ has begun to provide new insights into clinical, anatomical, and genetic characteristics relevant to glaucoma in a predominantly White-UK population. ${ }^{15-17}$

UK Biobank is a major UK health research resource, which aims to improve the prevention, diagnosis and treatment of a wide range of serious illnesses including cancer, heart diseases, stroke, diabetes, arthritis, osteoporosis, eye disorders, depression and dementia. UK Biobank recruited 502656 participants aged between 40-69 years in 2006-2010 from 22 centres across the UK. These people have undergone physical measures, provided biological samples, detailed information about themselves and have agreed to have their health status followed. Over many years this will build into a powerful resource to help scientists discover why some people develop particular diseases and others do not. ${ }^{18}$

In 2009, UK Biobank's international advisory committee recommended extending the scope of the study to include health measures, including an eye study module. Baseline vision, refraction, corneal biomechanics, and intraocular pressure (IOP) data are available on 133668 people, and spectral domain optical coherence tomography and fundus photos are available on 67321 people. ${ }^{19}$ Linkage to the wealth of health data in UK Biobank make this one the world's most powerful eye and vision research resources.

In this study, we aimed to explore the potential of UK Biobank to study the risk factors and clinical

characteristics of glaucoma in the UK, using self-reported diagnosis drawn from baseline questionnaires. We explored links between self-reported glaucoma and ocular, demographic, and socioeconomic factors.

\section{Materials and methods}

The UK Biobank study is a voluntary multisite crosssectional study of 502656 UK residents aged 40-69 years who were registered with the National Health Service living within 25 miles from any of the 22 study assessment centres. Nine million people registered with the UK National Health Service were invited to participate via mail, with study response rate of 5.5\%. Demographic and socioeconomic details were recorded for all participants. Ethnicity was identified by participants as either White, Chinese, Asian (in this context, typically Indian, Pakistani, or Bangladeshi, not Chinese or other east Asian descent), Black, or Mixed/ other. The Townsend deprivation index was used as a measure of deprivation. This Index has been validated for use in a UK-based population ${ }^{20}$, with higher scores representing greater levels of deprivation (range -6.258 to 9.643). Average household annual income before tax was also collected in the questionnaire. Education level and job type were not collected in a readily analyzable format, and thus have not been included in analysis. Self-reported glaucoma was based on those who selected 'glaucoma' from a predefined list of answers to the question 'Has a doctor ever told you that you have any of the following problems with your eyes?'

Four years after Biobank study began, additional funding was obtained to collect data on $\log$ MAR visual acuity, autorefraction, keratometry, IOP (Goldmanncorrected; Ocular Response Analyzer, Reichert, Depew, NY, USA) and corneal biomechanics at six-study assessment centers among 133959 eligible participants. Those who did not complete glaucoma question or who did not have valid IOP measurement in at least 1 eye were excluded, leaving a total of 112690 . The North West Multi-centre Research Ethics Committee approved the study (REC Reference Number: 06/MRE08/65), in accordance with the principles of the Declaration of Helsinki. Detailed information about the study is available at the UK Biobank website. ${ }^{18}$

Based on questionnaire responses, we estimated the frequency of self-reported diagnoses of glaucoma in this subset of 112690 UK Biobank participants. Relationships between demographic, socioeconomic and ocular variables with self-reported diagnoses of glaucoma were examined. Statistical analysis was performed using STATA version 12.0 (Statacorp LP, College Station, TX, USA). Values were considered significant if $P<0.01$.

\section{Results}

Among 112690 UK Biobank participants who completed ocular assessments, 1916 (1.7\%) reported a diagnosis of glaucoma. Baseline characteristics are reported in Table 1. Mean age for individuals reporting glaucoma was 61.4 years (SE 0.1, SD 6.2) compared with 56.7 (SE 0.2, SD 8.1) years for those without a glaucoma diagnosis (Table 1). The frequency of reported glaucoma significantly increased with older age and male gender (Table 1, Figure 1). Rates of glaucoma were $0.5 \%$ among those 40-49 years old, $1.2 \%$ among those 50-59 years old, and $2.7 \%$ among those 60-69 years old $(P<0.001$ using $40-49$ years of age as reference group; Figure 1). A total of $2.1 \%$ of men in the study population reported glaucoma compared with $1.4 \%$ of women $(P<0.001)$.

The frequency of self-reported glaucoma was significantly higher amongst Black and Asian participants than the baseline White ethnicity (Figure 2). A total of $3.3 \%$ of Black participants $(P<0.001)$ and $2.1 \%$ of Asian participants $(P=0.009)$ reported having glaucoma compared with $1.6 \%$ of the White population sampled (Figure 2). There was no significant difference in rates of self-reported glaucoma between Chinese or mixed/other ethnicities as compared with White participants. 
Table 1 Participant characteristics

\begin{tabular}{|c|c|c|}
\hline & No glaucoma & Glaucoma \\
\hline *Age & $56.7 \pm 0.02$ & $61.4 \pm 0.14$ \\
\hline${ }^{+}$Male gender & $45.6 \pm 0.1 \%$ & $56.6 \pm 1.1 \%$ \\
\hline \multicolumn{3}{|l|}{${ }^{+}$Ethnicity } \\
\hline White & $89.7 \pm 0.1 \%$ & $85.1 \pm 0.8 \%$ \\
\hline Chinese & $0.5 \pm 0.02 \%$ & $0.4 \pm 0.1 \%$ \\
\hline Asian & $3.8 \pm 0.1 \%$ & $4.8 \pm 0.5 \%$ \\
\hline Black & $3.4 \pm 0.1 \%$ & $6.8 \pm 0.6 \%$ \\
\hline Mixed/Other & $2.5 \pm 0.04 \%$ & $2.8 \pm 0.4 \%$ \\
\hline \multicolumn{3}{|l|}{${ }^{+}$Income } \\
\hline$<£ 18000$ & $21.7 \pm 0.1 \%$ & $31.9 \pm 1.2 \%$ \\
\hline$£ 18000-30999$ & $24.9 \pm 0.1 \%$ & $27.2 \pm 1.1 \%$ \\
\hline$£ 31000-51999$ & $25.7 \pm 0.1 \%$ & $22.3 \pm 1.0 \%$ \\
\hline$£ 52000-100000$ & $21.1 \pm 0.1 \%$ & $14.9 \pm 0.9 \%$ \\
\hline$>£ 100000$ & $6.5 \pm 0.1 \%$ & $3.6 \pm 0.5 \%$ \\
\hline *Townsend deprivation index & $-0.95 \pm 0.01$ & $-0.72 \pm 0.07$ \\
\hline
\end{tabular}

${ }^{*}$ Mean or ${ }^{+}$percentage \pm SE

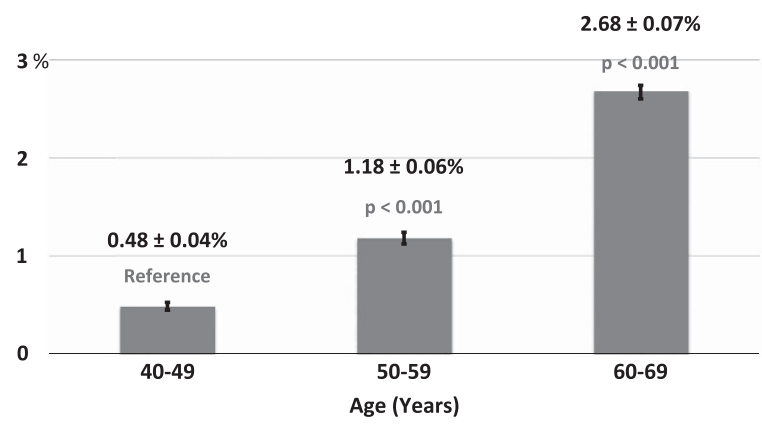

Figure 1 Frequency of self-reported glaucoma by age $(\% \pm \mathrm{SE})$. There is a significantly greater rate of glaucoma reported by older participants $(P<0.001)$.

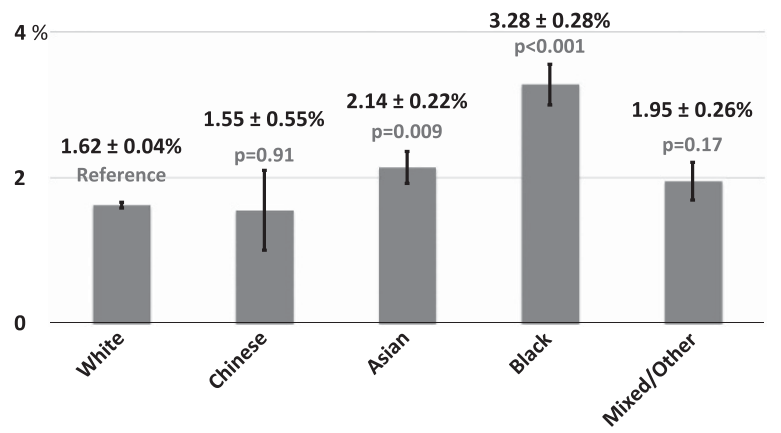

Figure 2 Reported rates of glaucoma by ethnicity ( $\% \pm \mathrm{SE})$. The rates of self-reported glaucoma is greatest in Black $(P<0.001)$ and Asian $(P=0.009)$ participants.

Mean Goldmann-corrected (18.26 mm Hg right eye, $18.01 \mathrm{~mm} \mathrm{Hg}$ left eye) and cornea-corrected IOP (18.87 $\mathrm{mm} \mathrm{Hg}$ right eye, $18.74 \mathrm{~mm} \mathrm{Hg}$ left eye) is greater in participants who report a diagnosis of glaucoma $(P<0.001$; Table 1). Both lower corneal hysteresis $(9.96$ right eye, 9.89 left eye, $P<0.001$ ) and reduced visual

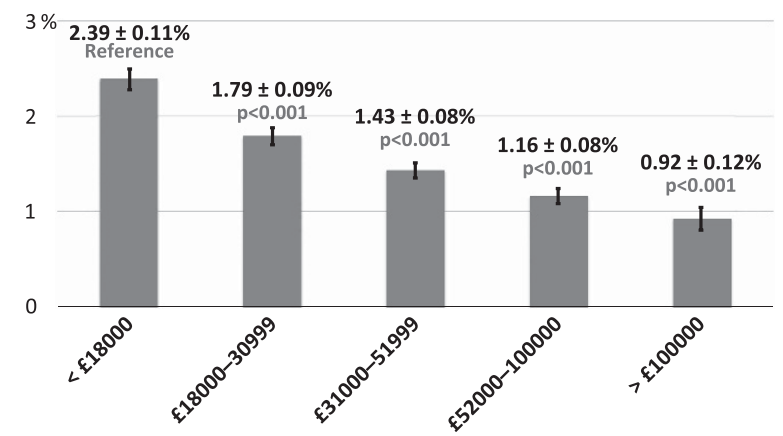

Figure 3 Frequency of self-reported glaucoma by annual income $(\% \pm \mathrm{SE})$. The rate of self-reported glaucoma is inversely related to income, and extends across the full range of the income spectrum.

Table 2 Multivariable regression analysis of risk factors for reported glaucoma

\begin{tabular}{|c|c|c|c|}
\hline & Odds ratio & $\begin{array}{c}95 \% \\
\text { confidence } \\
\text { interval }\end{array}$ & P-value \\
\hline \multicolumn{4}{|l|}{ Age, years } \\
\hline $40-49$ & Reference & Reference & Reference \\
\hline $50-59$ & 2.49 & $1.98 \quad 3.12$ & $<0.001$ \\
\hline $60-69$ & 5.41 & $4.37 \quad 6.70$ & $<0.001$ \\
\hline Male gender (vs female) & 1.50 & 1.351 .66 & $<0.001$ \\
\hline \multicolumn{4}{|l|}{ Ethnicity } \\
\hline White & Reference & Reference & Reference \\
\hline Chinese & 1.17 & $0.50 \quad 2.73$ & 0.72 \\
\hline Asian & 1.46 & 1.111 .91 & 0.006 \\
\hline Black & 2.81 & $2.24 \quad 3.51$ & $<0.001$ \\
\hline Mixed/other & 1.45 & $1.02 \quad 2.06$ & 0.04 \\
\hline \multicolumn{4}{|l|}{ Income } \\
\hline$<£ 18000$ & Reference & Reference & Reference \\
\hline$£ 18000-30999$ & 0.82 & $0.71 \quad 0.94$ & 0.003 \\
\hline$£ 31000-51999$ & 0.82 & $0.68 \quad 0.91$ & 0.001 \\
\hline$£ 52000-100000$ & 0.78 & 0.66 & 0.003 \\
\hline$>£ 100000$ & 0.64 & 0.48 & 0.003 \\
\hline IOPCC & 1.07 & 1.06 & $<0.001$ \\
\hline Visual acuity (per 0.1 logMar) & 1.08 & 1.06 & $<0.001$ \\
\hline
\end{tabular}

Abbreviation: IOPcc, cornea-corrected intraocular pressure in right eye. Visual acuity from right eye.

acuity (0.09 logMAR right eye, 0.08 logMAR left eye, $P<0.001)$ are significantly associated with self-reported glaucoma (Table 1).

As household income decreases, individuals are significantly more likely to report a diagnosis of glaucoma (Figure 3). Rates of glaucoma were highest among those of lowest annual income, $<£ 18000$ (2.4\%), and decreased as income increased, with the lowest rates among those with an income of $>£ 100000 /$ year $(0.9 \%, P<0.001$ between the highest and lowest groups glaucoma (Table 2)). Chi-squared testing for trend was significant at $P<0.001$. Those who report glaucoma have a higher mean 
Townsend deprivation index $(P<0.001$; Figure 4$)$. The Townsend deprivation index takes into account factors such as unemployment, non-car ownership, non-home ownership, and household overcrowding. ${ }^{20} \mathrm{~A}$ more positive score implies a higher degree of deprivation (UK average is score is 0 ).

Multivariable logistic regression modeling was performed with known risk factors for glaucoma (Table 3). There was a significant association with older

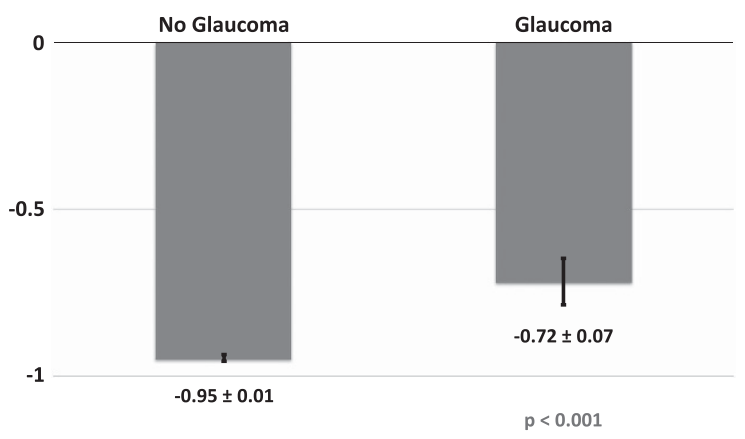

Figure 4 Comparison of Townsend deprivation index between individuals reporting a diagnosis of glaucoma vs those who do not (mean Townsend deprivation index $\pm S E$ ). Participants reporting glaucoma were significantly more likely to have a less-negative Townsend deprivation index score $(P<0.001)$. This indicates that both groups are less deprived than the UK average (index $=0$, scores $>0$ indicate relative deprivation), but those reporting a diagnosis of glaucoma were less likely to be as affluent as those without disease.

Table 3 Comparison of IOP, corneal biomechanics and visual acuity between participants reporting glaucoma and those who did not $($ mean \pm SE)

\begin{tabular}{|c|c|c|c|}
\hline & No glaucoma & Glaucoma & P-value \\
\hline \multicolumn{4}{|c|}{ IOP (mm Hg, Goldmann-corrected) } \\
\hline Right & $15.84 \pm 0.01$ & $18.26 \pm 0.12$ & $<0.001$ \\
\hline Left & $15.70 \pm 0.01$ & $18.01 \pm 0.12$ & $<0.001$ \\
\hline \multicolumn{4}{|c|}{ IOP (mm Hg, cornea-corrected) } \\
\hline Right & $16.02 \pm 0.01$ & $18.87 \pm 0.12$ & $<0.001$ \\
\hline Left & $15.95 \pm 0.01$ & $18.74 \pm 0.13$ & $<0.001$ \\
\hline \multicolumn{4}{|c|}{ Corneal hysteresis } \\
\hline Right & $10.67 \pm 0.01$ & $9.96 \pm 0.07$ & $<0.001$ \\
\hline Left & $10.63 \pm 0.01$ & $9.89 \pm 0.06$ & $<0.001$ \\
\hline \multicolumn{4}{|c|}{ Corneal resistance } \\
\hline Right & $10.74 \pm 0.01$ & $10.86 \pm 0.07$ & 0.03 \\
\hline Left & $10.66 \pm 0.01$ & $10.72 \pm 0.06$ & 0.30 \\
\hline \multicolumn{4}{|c|}{ Visual acuity (logMar) } \\
\hline Right & $0.025 \pm 0.001$ & $0.088 \pm 0.005$ & $<0.001$ \\
\hline Left & $0.021 \pm 0.001$ & $0.083 \pm 0.006$ & $<0.001$ \\
\hline
\end{tabular}

A self-reported diagnosis of glaucoma was significantly associated with greater IOP $(P<0.001)$, reduced corneal hysteresis $(P<0.001)$ and reduced visual acuity $(P<0.001)$. age (OR 2.49, 95\% CI 1.98-3.12 for ages 50-59 years and OR 5.41, 95\% CI 4.37-6.70 for ages 60-69 years, as compared with ages 40-49); male gender (OR 1.50, 95\% CI 1.35-1.66); Black or Asian ethnicity (OR 2.81, 95\% CI 2.24-3.81 and OR 1.46, 95\% CI 1.11-1.91, respectively, compared to Whites); high IOP (OR 1.07 per $\mathrm{mm} \mathrm{Hg}$, 95\% CI 1.06-1.08) and worse visual acuity (OR 1.08 per 0.1 $\log$ MAR, 95\% CI 1.06-1.11). Participants with a household annual gross income $>£ 18000$ were statistically less likely to report a diagnosis of glaucoma. Consistent with our findings for income, testing for trends in glaucoma rates by Townsend deprivation index shows a positive correlation, that is, the higher the deprivation index, the more like a participant is to report glaucoma $(P=0.014$, multivariable regression analysis $P=0.003$ for deprivation index $\geq 1.07$ ).

\section{Discussion}

In this study of a large community cohort of British people, those reporting glaucoma conformed to recognized epidemiological characteristics of those with primary open-angle glaucoma (POAG). Among people of European origin, primary open-angle glaucoma accounts for around $70-85 \%$ of all cases of glaucoma, hence risk factors for POAG would be expected to predominate as determinants of disease in a UK population. Self-reported glaucoma cases were older and more likely to be male than those without the diagnosis. The rate of self-reported glaucoma increased nonlinearly with age. Black and Asian people were significantly more likely to report a diagnosis of glaucoma than White participants. Among our study participants, the overall self-reported glaucoma rate was $1.7 \%$ in people aged $40-69$ years. This rate of disease appears lower than figures from population prevalence studies in European derived populations aged 40 years and older $(2.08 \%$ Baltimore Whites, USA; $2.0 \%$ Melbourne, Australia; 2.9\% Egna-Neumarkt, Italy), where participants were all examined for glaucoma $7,21,22$ It has been well established in population-based studies in industrialized nations that at least $50 \%$ of all glaucoma remains undiagnosed. ${ }^{10,23,24}$ One recent study in the US using NHANES data puts the proportion of undiagnosed disease at $78 \% .{ }^{25}$ We therefore expect the rate of selfreported glaucoma in UK Biobank to be lower than published population surveys owing to undiagnosed disease.

One of the most striking finding of our study was that, among UK Biobank participants, those who report a diagnosis of glaucoma were more likely to have a lower income and be from a relatively less affluent background. Participants in the lowest income group ( <£18 000/year) were significantly more likely to have self-reported glaucoma, compared with any other income category, 
after adjusting for age, sex, race, IOP, and visual acuity. The mean Townsend deprivation index was -0.72 for those reporting glaucoma and -0.95 for those without $(P<0.001)$. This indicates that both groups are less deprived than the UK average (index $=0$, scores $>0$ indicate relative deprivation), but those reporting a diagnosis of glaucoma were less likely to be as affluent as those without disease. Furthermore, we observed a gradient toward higher rates of glaucoma with decreasing income across the socioeconomic spectrum. Among a cohort who are less deprived than the UK average, the findings of reduced affluence among those reporting disease than in those who do not, and of a trend toward increased rate of disease across the spectrum of income groups are both novel, and important for understanding the mechanisms which drive the previously reported association between socioeconomic deprivation and late presentation of glaucoma.

Previous research has established that higher rates of glaucoma are seen in those at the lowest end of the socioeconomic spectrum, and that these people tend to present later, with more advanced disease, primarily owing to limited access to primary eye care services. Higher rates of glaucoma have been observed in both homeless and poor nonhomeless populations than in the general population of Los Angeles. ${ }^{26}$ In the UK, greater individual and area level deprivation are associated with late presentation of glaucoma. ${ }^{27-29}$ People presenting with advanced glaucoma are more likely to come from an underprivileged area and be of lower occupational class, to have no access to a car, to have left full time education at age 14 years or younger, and to be tenants rather than owner occupiers. ${ }^{27}$

The theory that later presentation is related to deprivation is apparently supported and explained by findings from the UK and Canada. A geographical mapping study in the UK identified a clear disparity between areas of deprivation and location of optometric services. ${ }^{28}$ In Canada, patients with newly diagnosed glaucoma were less likely to come from the poorest neighborhood areas (16\%, compared with an expected $20 \%, P=0.56)$. Compared with those from the poorest areas, people from the richest neighborhoods appeared to have a substantially lower risk for having moderate or advanced glaucoma at first presentation (prevalence ratio $0.66,95 \%$ confidence interval: $0.43-1.02, P=0.06)$. This association was stronger among those $\geq 65$ years old $(P=0.006)$. These findings may suggest that socioeconomic deprivation is associated with greater severity of glaucoma at presentation, and may indicate relative underdetection of glaucoma in poorer groups. ${ }^{30}$ Fraser et $a l^{27}$ also speculated that material deprivation may be associated with 'more aggressive' disease, as well as later presentation. Our findings may offer some support for this theory. There do not appear to be two groups (the 'deprived' and the 'wealthy') and certainly there does not appear to be a clear threshold at which the impact of deprivation is felt. The same pattern of a spectrum of increasing risk has been seen for all cause mortality, coronary heart disease mortality, and all cause vascular mortality across the range of deprivation (index of multiple deprivation) in a longitudinal study of British women. The authors commented that these relationships seemed to be mediated largely, although not exclusively, through health-related behavior such as physical activity, alcohol consumption, fruit and vegetable intake, and smoking. ${ }^{31}$

There is a clear relationship between deprivation and access to eye care services in both developing and developed world settings. In Pakistan, cataract surgical coverage was higher in affluent clusters $(80.6 \%)$ than in medium $(76.8 \%)$ and poor areas $(75.1 \%)$. Intraocular lens implantation rates were significantly lower in participants from poorer households. $10.2 \%$ of adults living in affluent clusters presented to the examination station wearing spectacles, compared with $6.7 \%$ in medium clusters and $4.4 \%$ in poor cluster areas. Spectacle coverage in affluent areas was more than double that in poor clusters $(23.5 \%$ vs $11.1 \%, P<0.001) .{ }^{32}$ In a cross-sectional sample representing the non-institutionalized US population aged $40+$, people with a low poverty-income ratio and an age-related eye disease were less likely to have visited an eye care provider $(62.7 \%$ vs $80.1 \%$; $P<0.001)$ or undergone a dilated eye examination in the past 12 months $(64.3 \%$ vs $80.4 \% ; P<0.001)$, compared with people at the higher end of the income spectrum, after adjustment for other factors. Similarly, persons with less than a high school education were less likely than those with at least a college education to report a visit to an eye care provider $(62.9 \%$ vs $80.8 \% ; P<0.001)$ or to have had a dilated eye examination $(64.8 \%$ vs $81.4 \% ; P<0.001) .{ }^{33}$ In addition, it has been shown that among patients presenting with a fractured neck of femur, binocular visual acuity is $<6 / 12$ in $46 \% .{ }^{34}$ Those who were visually impaired were more likely to have symptomatic visual complaints (58\% vs $26 \%$ ), but were less likely to be under optometric care (71\% vs $85 \%) .{ }^{34}$ A higher proportion of the group with visual impairment lived in areas of social deprivation (40\% vs $26 \%$ ). ${ }^{34}$ The majority of cases in this group of individuals are not in touch with ophthalmic services, leading the authors to comment that social deprivation appeared to be associated with the inability to access ophthalmic care. ${ }^{34}$

In the UK, the national healthcare system provides the majority of care at little or no charge. However, barriers to accessing regular eye examinations may still exist, including poor knowledge of eye health, concerns about the cost of spectacles, mistrust of optometrists and limited 
geographical access in socioeconomically deprived areas. These are believed to result in low uptake of services, and subsequent late presentation to ophthalmology clinics. ${ }^{35}$ Day et $a l^{28}$ suggested that it may not be feasible to rely on private 'high street' optometrists to provide primary eye care services for the most deprived. There have been calls for noncommercial primary eye care in the form of ophthalmic or optometric community services to provide primary eye care in socioeconomically deprived areas.

Funding would be required from sources other than eye examination fees and spectacle sales. In order to promote greater access to care, there have been calls for a significant shift of activity from secondary to primary care locations. It has been suggested that costs incurred could be offset by the utility gain from earlier detection of preventable sight loss. ${ }^{35}$

Limitations of this study clearly include the use of selfreported disease status, which can result in misclassification error. Participants with ocular hypertension or suspect glaucoma may mistakenly report a diagnosis of glaucoma. There will be underascertainment of people with disease, as around $50 \%$ of all cases will not have been diagnosed and therefore will not be captured. The overall direction of impact of these potential errors is unknown. However, we believe the overall pattern seen in the data would not be diminished. On the contrary, if people from more deprived background have greater barriers to health seeking behavior and care, one would expect that they would be less likely to be aware of their diagnosis and not report it, and probably less likely to attend health research activities such as UK Biobank. We believe it is likely the true trends identified here for socioeconomics influencing the risk of glaucoma would be accentuated, if the true burden of disease were identified. We were not able to differentiate between POAG, angle-closure glaucoma and secondary disease, and hence are not able to comment on the relative impact of socioeconomics on different forms of glaucoma. It has been reported that acute primary angle-closure risk is higher in people from deprived backgrounds. ${ }^{36}$ Finally, as UK Biobank has a low response rate of $5.5 \%$, figures for rates of disease must be treated with caution. The low participation rate limits external validity and is not representative of the UK population. However, the study size of 112690 is a major strength that allows us to detect and quantify small effects. We also used standardized techniques, and included individuals from several different ethnicities.

In the UK Biobank cohort, the distribution of selfreported glaucoma shows expected associations with higher rates of disease in the elderly, men, and Black and Asian people. As a novel finding, we have identified an inverse relationship between self-reported glaucoma and measures of socioeconomic status. The cohort is of a higher socioeconomic status than the average members of the UK population. The relationship between income category and rate of self-reported glaucoma showed a clear trend of increasing disease across the full spectrum of income. These two facts give an important new perspective to views on the relationship between glaucoma and socioeconomics. There are undoubtedly barriers to eye care for detection and treatment. However, studies of mortality point towards the effects of deprivation being mediated through 'health-related behaviors' such as physical activity, alcohol consumption, fruit and vegetable intake, and smoking.

We now plan to examine structural biomarkers for glaucoma in the UK Biobank cohort (such as retinal nerve fiber layer thickness on OCT examinations) and assess firstly if the socioeconomic relationship exists with evidence of end organ damage, and secondly to use data soon to become available, such as the serum biomarker panel, diet, and physical exercise data, on this cohort to try to determine the biological mechanisms, which underlie the novel findings reported here.

\section{Summary}

\section{What was known before}

- Patients with glaucoma from lower socioeconomic backgrounds present with more advanced disease.

- Higher rates of glaucoma have been reported in people suffering extreme deprivation (eg, homelessness).

- These factors have been linked to barriers to accessing care.

What this study adds

- In a large UK cohort that is more affluent than the UK average, people reporting a diagnosis of glaucoma are from a less wealthy background, and show more indices of relative deprivation.

- The rate of glaucoma is inversely related to income, and this relationship extends across the full range of income and deprivation categories.

- Similar trends in studies of mortality have been linked to health-related behavior including exercise, smoking, alcohol consumption and fruit and vegetables in the diet.

\section{Conflict of interest}

The authors declare no conflict of interest.

\section{Acknowledgements}

We acknowledge a proportion of our financial support from the UK Department of Health through an award made by the National Institute for Health Research to Moorfields Eye Hospital NHS Foundation Trust and UCL Institute of Ophthalmology for a Biomedical Research 
Centre for Ophthalmology. The views expressed in this publication are those of the authors and not necessarily those of the UK Department of Health. Miss Chan holds a PhD studentship supported by the Medical Research Council (London) and Royal College of Ophthalmologists (London), and an International Glaucoma Association award. Dr Ko is supported by ORS and GRS scholarships from University College London. Dr Ko, Mr Strouthidis, and Professor Foster are supported by a grant from the International Glaucoma Association (London). Professor Foster is supported by funding from the Richard Desmond Charitable Trust via Fight for Sight (London). Mr Patel, Ms Muthy, Professor Khaw, Mr Strouthidis and Professor Foster are supported by grants from the NIHR Biomedical Research Centre at Moorfields Eye Hospital and the UCL Institute of Ophthalmology.

\section{Members of the UK Biobank Eye and Vision Consortium}

Prof Tariq Aslam, Dr Sarah Barman, Prof Paul Bishop, Mr Peter Blows, Dr Catey Bunce, Dr Roxana Carare, Prof Usha Chakravarthy, Miss Michelle Chan, Mrs Antonietta Chianca, Dr Valentina Cipriani, Prof David Crabb, Mrs Philippa Cumberland, Dr Alexander Day, Miss Parul Desai, Prof Bal Dhillon, Prof Andrew Dick, Prof Paul Foster, Dr John Gallacher, Prof David (Ted) Garway-Heath, Mr Srini Goverdhan, Prof Jeremy Guggenheim, Mrs Priyal Gupta, Prof Chris Hammond, Dr Ruth Hogg, Prof Anne Hughes, Mr Pearse Keane, Prof Sir Peng Tee Khaw, Mr Anthony Khawaja, Mr Gerassimos Lascaratos, Prof Andrew Lotery, Prof Phil Luthert, Dr Tom MacGillivray, Dr Sarah Mackie, Prof Keith Martin, Ms Michelle McGaughey, Dr Bernadette McGuinness, Dr Gareth McKay, Mr Martin McKibbin, Dr Danny Mitry, Prof Tony Moore, Prof James Morgan, Ms Zaynah Muthy, Mr Eoin O'Sullivan, Dr Chris Owen, Mr Praveen Patel, Dr Tunde Peto, Prof Jugnoo Rahi, Dr Alicja Rudnicka, Miss Carlota Grossi Sampedro, Mr David Steel, Mrs Irene Stratton, Mr Nicholas Strouthidis, Prof Cathie Sudlow, Dr Caroline Thaung, Miss Dhanes Thomas, Prof Emanuele Trucco, Mr Adnan Tufail, Prof Stephen Vernon, Mr Ananth Viswanathan, Miss Cathy Williams, Dr Katie Williams, Prof John Yates, Dr Max Yates, Dr Jennifer Yip, Dr Haogang Zhu.

\section{References}

1 Pascolini D, Mariotti SP. Global estimates of visual impairment: 2010. Br J Ophthalmol 2012; 96: 614-618.

2 Bunce C, Wormald R. Leading causes of certification for blindness and partial sight in England \& Wales. BMC Public Health 2006; 6: 58
3 Quigley HA, Broman AT. The number of people with glaucoma worldwide in 2010 and 2020. Br J Ophthalmol 2006; 90: 262-267.

4 Kotecha A, Turner S, Vasilakis C, Utley M, Fulop N, AzuaraBlanco A et al. Improving care and increasing efficiencychallenges in the care of chronic eye diseases. Eye (Lond) 2014; 28: 779-783.

5 Shah S, Murdoch IE. NICE - impact on glaucoma case detection. Ophthalmic Physiol Opt 2011; 31: 339-342.

6 De Silva SR, Riaz Y, Purbrick RMJ, Salmon JF. There is a trend for the diagnosis of glaucoma to be made at an earlier stage in 2010 compared to 2008 in Oxford, United Kingdom. Ophthalmic Physiol Opt 2013; 33: 179-182.

7 Tielsch JM, Sommer A, Katz J, Royall RM, Quigley HA, Javitt $\mathrm{J}$. Racial variations in the prevalence of primary open-angle glaucoma. The Baltimore Eye Survey. JAMA 1991; 266: 369-374.

8 Sommer A, Tielsch JM, Katz J, Quigley HA, Gottsch JD, Javitt J et al. Relationship between intraocular pressure and primary open angle glaucoma among White and Black Americans. Arch Ophthalmol 1991; 109: 1090-1095.

9 Klein B, Klein R, Sponsel WE, Franke T, Cantor LB, Martone $\mathrm{J}$ et al. Prevalence of glaucoma. The Beaver Dam Eye Study. Ophthalmology 1992; 99: 1499-1504.

10 Mitchell P, Smith W, Attebo K, Healey P. Prevalence of openangle glaucoma in Australia. The Blue Mountains Eye Study. Ophthalmology 1996; 103: 1661-1669.

11 De Voogd S, Ikram MK, Wolfs RC, Jansonius NM, Hofman A, de Jong PT. Incidence of open-angle glaucoma in a general elderly population: The Rotterdam Study. Ophthalmology 2005; 112: 1487-1493.

12 Hollows FC, Graham PA. Intra-ocular pressure, glaucoma, and glaucoma suspects in a defined population. $\mathrm{Br} J$ Ophthalmol 1966; 50: 570-586.

13 Bankes J, Perkins E, Tsolakis S, Wright J. Bedford glaucoma survey. Br Med J 1968; 1: 791.

14 Khawaja AP, Chan MP, Hayat S, Broadway DC, Luben R, Garway-Heath DF et al. The EPIC-Norfolk Eye Study: rationale, methods and a cross-sectional analysis of visual impairment in a population-based cohort. BMJ Open 2013; 3: e002684

15 Foster PJ, Broadway DC, Garway-Heath DF, Yip JL, Luben $\mathrm{R}$, Hayat $\mathrm{S}$ et al. Intraocular pressure and corneal biomechanics in an adult British population: The EPICNorfolk eye study. Invest Ophthalmol Vis Sci 2011; 52: 8179-8185.

16 Khawaja AP, Chan MP, Garway-Heath DF, Broadway DC, Luben R, Sherwin JC et al. Associations with retinal nerve fiber layer measures in the EPIC-Norfolk eye study. Invest Ophthalmol Vis Sci 2013; 54: 5028-5034.

17 Strange A, Bellenguez C, Sim X, Luben R, Hysi PG, Ramdas WD et al. Genome-wide association study of intraocular pressure identifies the GLCCI1/ICA1 region as a glaucoma susceptibility locus. Hum Mol Genet 2013; 22: 4653-4660.

18 Biobank http://www.ukbiobank.ac.uk/. Accessed on 18 May 2015.

19 UK Biobank Eye and Vision Consortium http://www. ukbiobankeyeconsortium.org.uk/. Accessed on 18 May 2015.

20 Townsend P. Poverty in the United Kingdom. Allen Lane and Penguin Books: London, UK, 1979.

21 Bonomi L, Marchini G, Marraffa M, Bernardi P, De Franco I, Perfetti $\mathrm{S}$ et al. Prevalence of glaucoma and intraocular 
pressure distribution in a defined population. Ophthalmology 1998; 105: 209-215.

22 Wensor M, McCarty C, Stanislavsky Y, Livingston P, Taylor $\mathrm{H}$. The prevalence of glaucoma in the Melbourne Visual Impairment Project. Ophthalmology 1998; 105: 733-739.

23 Dielemans I, Vingerling JR, Wolfs RC, Hofman A, Grobbee DE, de Jong PT. The prevalence of primary open-angle glaucoma in a population-based study in The Netherlands. The Rotterdam Study. Ophthalmology 1011994; 1851-1855.

24 Topouzis F, Wilson MR, Harris A, Anastasopoulos E, Yu F, Mavroudis L et al. Prevalence of open-angle glaucoma in Greece: The Thessaloniki Eye Study. Am J Ophthalmol 2007; 144: 511-520.

25 Shaikh Y, Yu F, Coleman AL. Burden of Undetected and Untreated Glaucoma in the United States. Am J Ophthalmol 2014; 158: 1121-1129.e1.

26 Ho J, Chang R, Wheeler N, Lee D. Ophthalmic disorders among the homeless and non-homeless in Los Angeles. J Am Optom Assoc 1997; 68: 567-573.

27 Fraser S, Bunce C, Wormald R, Brunner E. Deprivation and late presentation of glaucoma: case-control study. BMJ 2001; 322: 639-643.

28 Day F, Buchan JC, Cassells-Brown A, Fear J, Dixon R, Wood F. A glaucoma equity profile: correlating disease distribution with service provision and uptake in a population in Northern England, UK. Eye (Lond) 2010; 24: 1478-1485.

29 Ng WS, Agarwal PK, Sidiki S, McKay L, Townend J, Azuara-Blanco A. The effect of socio-economic deprivation on severity of glaucoma at presentation. $\mathrm{Br} J$ Ophthalmol 2010; 94: 85-87.

30 Buys YM, Jin YP. Canadian Glaucoma Risk Factor Study Group. Socioeconomic status as a risk factor for late presentation of glaucoma in Canada. Can J Ophthalmol 2013; 48: 83-87.

31 Sánchez-Santos MT, Mesa-Frias M, Choi M, Nüesch E, Asunsolo-Del Barco A, Amuzu A et al. Area-Level Deprivation and Overall and Cause-Specific Mortality: 12
Years' Observation on British Women and Systematic Review of Prospective Studies. PLoS One 2013; 8: e72656.

32 Gilbert CE, Shah SP, Jadoon MZ, Bourne R, Dineen B, Khan MA et al. Poverty and blindness in Pakistan: results from the Pakistan national blindness and visual impairment survey. BMJ 2008; 336: 29-32.

33 Zhang X, Beckles GL, Chou CF, Saaddine JB, Wilson MR, Lee PP et al. Socioeconomic Disparity in Use of Eye Care Services Among US Adults With Age-Related Eye Diseases: National Health Interview Survey, 2002 and 2008. JAMA Ophthalmol 1312013; 1198-1206.

34 Cox A, Blaikie A, MacEwen CJ, Jones D, Thompson K, Holding D et al. Visual impairment in elderly patients with hip fracture: causes and associations. Eye (Lond) 2005; 19: 652-656.

35 Shickle D, Todkill D, Chisholm C, Rughani S, Griffin M, Cassels-Brown A et al. Addressing inequalities in eye health with subsidies and increased fees for General Ophthalmic Services in socio-economically deprived communities: a sensitivity analysis. Public Health 2015; 129: 131-137.

36 Nessim M, Denniston AK, Nolan W, Holder R, Shah P. Research into Glaucoma and Ethnicity (ReGAE) 8: is there a relationship between social deprivation and acute primary angle closure? Br J Ophthalmol 2010; 94: 1304-1306.

(i) This work is licensed under a Creative Commons Attribution 4.0 International License. The images or other third party material in this article are included in the article's Creative Commons license, unless indicated otherwise in the credit line; if the material is not included under the Creative Commons license, users will need to obtain permission from the license holder to reproduce the material. To view a copy of this license, visit http://creativecommons.org/licenses/by/ $4.0 /$ 